\title{
A characterization of pre-near-standardness in locally convex linear topological spaces
}

\section{J.J.M. Chadwick and R.W. Cross}

Let $X$ be a locally convex linear topological space. A point $z$ in an ultralimit enlargement of $X$ is pre-near-standard if and only it is finite and for every equicontinuous subset $S^{\prime}$ of the dual space $X^{\prime}$, a point $z^{\prime}$ belongs to * $S^{\prime} \cap \mu_{\sigma(X, X)}(0)$ only if $z^{\prime}(z)$ is infinitesimal.

\section{Introduction}

In a recent paper Luxemburg [2] obtains a characterization of pre-near-standardness for normed spaces leading to several interesting applications in the standard theory. Our object here will be to derive a characterization for locally convex spaces generalizing ([2], 3.17.2). An introduction to the theory of non-standard analysis can be found in [3] and [4]. In addition we make implicit use of ultralimit (or suitably saturated) enlargements. All the necessary metamathematical background will be found in [2]. For the topological vector space concepts see for example köthe [1].

Some definitions and notations will now be given. Let $\Omega$ be a family of subsets of a given set. We denote the (intersection) monad of $\Omega$ by $\mu(\Omega)$ (see [2]). By definition, $\mu(\Omega)=\cap\left({ }^{*} E: E \in \Omega\right)$.

When $\Omega$ is the filter $F$ of neighbourhoods of a point $x$ in a topological space $(X, \tau)$, we often write $\mu_{\tau}(x)$ for $\mu(F)$. The definition of $\mu_{\tau}(x)$ accords with that of Robinson [4]. 
If $(X, \tau)$ is a linear topological space, and $\left\{N_{\delta}: \delta \in \Delta\right\}$ the set of all neighbourhoods of the origin, then the set of vicinities $V_{\delta}=\left\{(x, y): x-y \in N_{\delta}\right\}$ is a uniformity $u$ on $X$ which induces the topology $\tau$. For any point $z \in{ }^{*} X$ we define

$$
\mu_{u}(z)=n(* V(z): V \in u) \text {, }
$$

where ${ }^{*} V(z)=\left({ }^{*}\right)(z)=\left\{x: x-z \in{ }^{*} V\right\}$.

We recall that a proper filter $F$ of subsets of $X$ is a Cauchy fizter if for each $V \in u$ there exists $F \in F$ such that $F \times F \subset V$, or equivalently, if and only if $\mu(F) \times \mu(F) \subset \mu(u)$. A Cauchy filter is called minimal if it does not properly contain a Cauchy filter.

The point $z \in{ }^{*} X$ is called pre-near-standard if there exists a filter $F$ of subsets of $X$ (necessarily a Cauchy filter) such that $\mu(F) \subset \mu_{u}(z)$. Clearly every near-standard point is pre-near-standard. The concept of pre-near-standardness was first introduced by Luxemburg [2]. A necessary and sufficient condition that a uniform space $A$ be precompact is that every point of $* A$ be pre-near-standard [2].

We shall make use of the following result:

THEOREM 1.1 ([2], 3.12.1). A point $z \in * X$ is pre-near-standard if and only if there exists a minimal cauchy filter $F$ such that $\mu(F)=\mu_{u}(z)$.

Throughout the remainder of this paper the following notation will be used. Except where stated otherwise, $X$ will denote a locally convex linear topological space whose generating family of seminorms is $\left(p_{\lambda}: \lambda \in \Lambda\right) \cdot X_{\lambda}$ will denote the seminormed space $\left(X, p_{\lambda}\right)$, and $S_{\lambda}^{\prime}$ the unit ball of the topological dual $X_{\lambda}^{\prime}$ of $X_{\lambda}$. For an arbitrary topological space $(X, \tau), \mu_{\tau}(x)$ denotes the $\tau$-monad of $x \in X$. In particular, $\mu_{d}(x)$ is the monad of $x$ in the discrete topology, and $\mu_{\sigma\left(X^{\prime}, X\right)}(0)$ is the monad of the origin of $X^{\prime}$ in its weak star topology. For any pair of real numbers $a, b$ the relation $a={ }_{1} b$ means that $a-b$ is infinitesimal. 
A point $z \epsilon^{*} X$ will be called finite if $p_{\lambda}(z)$ is finite for every standard $\lambda$ in $\Lambda$.

\section{The main theorem}

Our principal result will be the following:

THEOREM 2.1. Let $z$ be a point of $* X$. Then $z$ is pre-near-standard if and only if it is finite and for every equicontinuous set $S^{\prime}$ in $X^{\prime}, z^{\prime} \in{ }^{*} S^{\prime} \cap \mu_{\sigma\left(X^{\prime}, X\right)}(0)$ implies $z^{\prime}(z)={ }_{1} 0$.

The theorem will be proved by means of some auxiliary theorems and lemmas.

Let $z \in{ }^{*} X$ and let $B(z)$ denote the collection of all finite intersections of sets $\left\{x: p_{\lambda}(z-x)<\varepsilon\right\}$ where $\lambda$ and $\varepsilon>0$ are both standard.

THEOREM 2.2. The point $z$ is pre-near-standard if and only if each $B$ in $B(z)$ contains a standard point.

Proof. Suppose $z$ is pre-near-standard. It is clear that $\mu_{u}(z)=\left\{x: p_{\lambda}(x-z)=10\right.$ for all standard $\left.\lambda\right\}$. By Theorem 1.1, $\mu_{u}(z)$ is a filter monad and therefore $\mu_{d}(z) \subset \mu_{u}(z)$. Let $B \in B(z)$. Then $B$ is by definition internal and $\mu_{u}(z) \subset B$. Hence, since $\mu_{d}(z) \subset B, B$ contains a standard point by $([2], 2.8 .1)$.

Conversely, suppose that each member of $B(z)$ contains a standard point. For each $B \in B(z)$ select a standard $x_{B} \in B$. Now $B(z)$ becomes a directed set if we define $B_{1} \leq B_{2} \Longleftrightarrow B_{2} \subset B_{1}$ and then $\left(x_{B}: B \in B(z)\right)$ becomes a net. Let $F$ be the associated filter of subsets $\left\{x_{B^{\prime}}: B^{\prime} \geq B\right\}$.

We will show that $F$ is a Cauchy filter. We have

$$
\mu(F)=\left\{x_{B}: B \in{ }^{*} B(z), B \geq B^{\prime} \text { for all standard } B^{\prime}\right\} \text {. }
$$

Therefore 


$$
\begin{aligned}
x \in \mu(F) & \Rightarrow x \in B \text { for every standard } B \text { in } \star B(z) \\
& \Rightarrow p_{\lambda}(x-z)<\varepsilon \text { for all standard } \lambda \text { and } \varepsilon>0 \\
& \Rightarrow p_{\lambda}(x-z)=10 \text { for all standard } \lambda .
\end{aligned}
$$

Hence if $x, y \in \mu(F)$ we have

$$
p_{\lambda}(x-y) \leq p_{\lambda}(x-z)+p_{\lambda}(z-y)==_{1} 0,
$$

whence $(x, y) \in \mu(u)$. Consequently $\mu(F) \times \mu(F) \subset \mu(u)$, that is, $F$ is Cauchy.

Now $x \in \mu(F) \Rightarrow p_{\lambda}(x-z)=10$ for all standard $\lambda \Rightarrow x \in \mu_{u}(z)$. Thus $\mu(F) \subset \mu_{u}(z)$ showing that $z$ is pre-near-standard.

We remark that Theorem 2.2 implies ([2], 3.17.1).

COROLLARY 2.3. Every pre-near-standard point is finite.

PROPOSITION 2.4. If $z \in{ }^{*} X$ is finite, then $f(z)$ is also finite for every standard $f \in X^{\prime}$.

Proof. Let $z \in{ }^{*} X$ be finite, and let $f \in X^{\prime}$. If $f(z)$ is infinite, then for all standard $\lambda, p_{\lambda}(z / f(z))==_{1} 0$ since $p_{\lambda}(z)$ is finite. Thus $z / f(z) \in \mu_{\tau}(0)$. But $f(z / f(z))=1$, contradicting the continuity of $f,([3], 5.4 .1)$.

DEFINITION 2.5. Let $z$ be a finite point of " $X$. The functional $s t_{w}(z),(c f .[2], p .83)$, is defined by

$$
\text { st }_{w}(z)(f)=\operatorname{stf}(z) \text { for } f \in X^{\prime} \text {, }
$$

where stf(z) denotes the standard part of $f(z),([4]$, p. 57). By Proposition 2.4, st ${ }_{w}(z)$ is a linear functional on $X^{\prime}$.

We make use of the following elementary result:

LEMMA 2.6. Let $S^{\prime}$ be a uniform space, and $\left(g_{\delta}: \delta \in \Delta\right)$ a net of continuous mappings of $S^{\prime}$ into the space of real numbers such that $g_{\delta} \rightarrow g$ uniformly on $S^{\prime}$. Then $g$ is continuous.

THEOREM 2.7. If $z \in{ }^{*} X$ is pre-near-standard, then st $_{\omega}(z)$ is 
continuous in the $\sigma\left(X^{\prime}, X\right)$ topology on every equicontinuous subset of $X^{\prime}$.

Proof. Let $z \in \epsilon^{*} X$ be pre-near-standard and let $S^{\prime}$ be an equicontinuous subset of $X^{\prime}$. By Theorem 2.2, for each $B$ in $B(z)$ we can select a standard $x_{B}$ in $B$. Then $\hat{x}_{B}$ is $\sigma\left(X^{\prime}, X\right)$ continuous on $S^{\prime}$. By Lemma 2.6 it is sufficient to show that $\hat{x}_{B}$ converges uniformly to $\operatorname{st}_{w}(z)$ on $S^{\prime}$. This will follow if we can show that for given $\varepsilon>0$, there exists $B \in B(z)$ such that

$$
B^{\prime} \subset B \Rightarrow\left|\hat{x}_{B^{\prime}}(f)-s t_{w}(z)(f)\right|<\varepsilon \text { for all } f \in S^{\prime} .
$$

Let $N$ be a O-neighbourhood such that

$$
x-y \in N=|f(x)-f(y)|<\varepsilon / 2 \text { for all } f \in S^{\prime}
$$

and such that $N$ contains an element $B_{0}$ of $B(0)$. Set

$z+B_{0}=B \in B(z)$. Then for all $B^{\prime} \subset B, x_{B^{\prime-z} \in B_{0} \subset{ }^{*}}$, whence $\left|f\left(x_{B^{\prime}}\right)-f(z)\right|<\varepsilon / 2 \quad\left(f \in S^{\prime}\right)$. Consequently $\left|\hat{x}_{B}(f)-s t_{w}(z)(f)\right|=\left|f\left(x_{B}\right)-s t f(z)\right| \leq$

$$
\left|f\left(x_{B^{\prime}}\right)-f(z)\right|+|f(z)-\operatorname{stf}(z)| \leq_{1} \varepsilon / 2<\varepsilon,
$$

as required.

THEOREM 2.8. Let $z \in{ }^{*} X$ be pre-near-standard, and let $S^{\prime}$ be an equicontinuous subset of $X^{\prime}$. Then $s t_{w}(z)\left(z^{\prime}\right)={ }_{1} z^{\prime}(z)$ for every $z^{\prime}$ in ' $S$ '.

Proof. Let $z$ and $S^{\prime}$ be as stated, and let $\varepsilon>0$ be given. Since $S^{\prime}$ is equicontinuous, there is a basic O-neighbourhood $N$ in $X$ such that

$$
y \in N=\left|x^{\prime}(y)\right|<\dot{\varepsilon} / 2 \quad\left(x^{\prime} \in S^{\prime}\right) .
$$

Then $\left|z^{\prime}(y)\right|<\varepsilon / 2$ for all $z^{\prime}$ in ${ }^{*} S^{\prime}$ and $y$ in ${ }^{*} N$. Next we have for $z^{\prime} \in{ }^{*} S^{\prime}$

$$
\text { st }_{w} z\left(z^{\prime}\right)-z^{\prime}(z)=\mathrm{st}_{w}(z-x)\left(z^{\prime}\right)+\mathrm{st}_{w} x\left(z^{\prime}\right)-z^{\prime}(z-x)-z^{\prime}(x)
$$

whenever $x$ is standard. 
Put $B=\{z\}-{ }^{*} N$. Since $z$ is pre-near-standard we can select $x \in X$ with $x \in B$ (Theorem 2.2). Putting $y=z-x, y$ belongs to *N and so by (1), $\left|x^{\prime}(y)\right|<\varepsilon / 2 \quad\left(x^{\prime} \in S^{\prime}\right)$. Hence for (standard) $x^{\prime} \in S^{\prime}$,

$$
\mid \text { st }_{w}(z-x)\left(x^{\prime}\right)|=| \operatorname{stx}^{\prime}(z-x)\left|={ }_{1}\right| x^{\prime}(y) \mid<\varepsilon / 2 \text {. }
$$

Transferring to $* X^{\prime}$ we obtain

$$
\left|\mathrm{st}_{w}(z-x)\left(z^{\prime}\right)\right|<\varepsilon / 2 \quad\left(z^{\prime} \in{ }^{*} S^{\prime}\right) .
$$

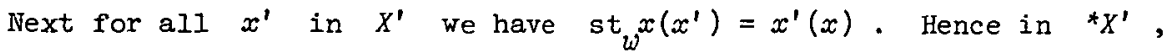

$$
\text { st } w^{x\left(z^{\prime}\right)=z^{\prime}(x)} \text {. }
$$

Since $z-x \in{ }^{*} N$, we have from (1)

$$
\left|z^{\prime}(z-x)\right|<\varepsilon / 2 \quad\left(z^{\prime} \in{ }^{*} S^{\prime}\right) .
$$

Finally,

$$
\begin{array}{r}
\left|\mathrm{st}_{w} z\left(z^{\prime}\right)-z^{\prime}(z)\right| \leq\left|\mathrm{st}_{w}(z-x)\left(z^{\prime}\right)\right|+\left|s t_{w} x\left(z^{\prime}\right)-z^{\prime}(x)\right|+\left|z^{\prime}(z-x)\right|<\varepsilon \\
\text { by (2), (3) and (4). }
\end{array}
$$

It follows that $\mathrm{st}_{w} z\left(z^{\prime}\right)={ }_{1} z^{\prime}(z)$ for all $z^{\prime} \epsilon^{\star} S^{\prime}$.

PROPOSITION 2.9. Suppose $S^{\prime}$ is an equicontinuous subset of $X^{\prime}$ and $\boldsymbol{z}$ any finite point of ${ }^{*} X$ such that

(i) st $_{w^{2}} z\left(z^{\prime}\right)==_{1} z^{\prime}(z) \quad\left(z^{\prime} \in{ }^{*} S^{\prime}\right)$;

(ii) st $w^{z}$ is $\sigma\left(X^{\prime}, X\right)$ continuous on $S^{\prime}$.

Then $z^{\prime}(z)={ }_{1} 0$ for all $z^{\prime} \epsilon{ }^{*} S^{\prime} \cap \mu_{\sigma\left(X^{\prime}, X\right)}(0)$.

Proof. We have only to note that since st ${ }_{w}(z)$ is $\sigma\left(X^{\prime}, X\right)$ continuous on $S^{\prime}$,

$$
\text { st }_{w} z\left(\mu_{\sigma\left(X^{\prime}, X\right)}(0) \cap{ }^{*} S^{\prime}\right) \subset \mu \text { st }_{w} z(0)=\mu(0)
$$

by Robinson's continuity criterion ([4], p. 98). Consequently $z^{\prime} \in \mu_{\sigma\left(X^{\prime}, X\right)}(0) \cap * S^{\prime}=s_{w^{\prime}} z\left(z^{\prime}\right)={ }_{1} 0$ and the result follows on applying condition (i).

Let us observe that $X_{\lambda}^{\prime}$ is embeddable as a linear subspace of $X^{\prime}$. 
The following lemma is easily verified:

LEMMA 2.10. $S_{\lambda}^{\prime}$ is an equicontinuous subset of $X^{\prime}$.

THEOREM 2.11. Let $z \in{ }^{*} X$. Then $z$ is pre-near-standard if and only if $z$ is pre-near-standard in each ${ }^{*} X_{\lambda}$.

Proof. Let $z \in{ }^{*} X$ be pre-near-standard. By Theorem 2.2, the set $\left\{x: p_{\lambda}(x-z)<\varepsilon\right\}$ contains a standard point, and hence $z$ is pre-near-standard in ${ }^{*} X_{\lambda}$.

Conversely, suppose that $z$ is pre-near-standard in each ${ }^{*} X_{\lambda}$. By Theorem 1.1, there exists a minimal Cauchy filter $F_{\lambda}$ such that $\mu_{u_{\lambda}}(z)=\mu\left(F_{\lambda}\right)$ where $u_{\lambda}$ is the uniformity of $\left(X, p_{\lambda}\right)$. Since $z \in \mu\left(F_{\lambda}\right)$ for each $\lambda$, it follows that $\cap_{\mu}\left(F_{\lambda}\right) \neq \varnothing$, and so the union filter $F=\bigvee F_{\lambda}$ exists as the filter of all finite intersections $F_{\lambda_{1}} \cap \ldots \cap F_{\lambda_{n}}$, where $F_{\lambda_{i}} \in F_{\lambda_{i}}$. Also

$$
\mu(F)=n\left(\mu\left(F_{\lambda}\right): \lambda \in \Lambda\right) \text {. }
$$

(a) $\mu(u)=n \mu\left(u_{\lambda}\right)$.

It suffices to show that $u=\bigvee u_{\lambda}$. Indeed the uniformity $u$ is generated by sets of the form

$$
\begin{aligned}
B=\left\{(x, y): x-y \in \bigcap_{i=1}^{n}\left\{p_{\lambda_{i}}<\varepsilon_{i}\right\}\right\}= & \\
& \prod_{i=1}^{n}\left\{(x, y): x-y \in\left\{p_{\lambda_{i}}<\varepsilon_{i}\right\}\right\}=\bigcap_{i=1}^{n} B_{\lambda_{i}},
\end{aligned}
$$

say. But the $B_{\lambda_{i}}$ form a base for $u_{\lambda_{i}}$, so (a) follows from the definition of $\bigvee u_{\lambda_{i}}$.

(b) $\mu_{u}(z)=\cap \mu_{u_{\lambda}}(z)$. 
We have

$$
\begin{aligned}
\mu_{u}(z) & =\mu(u)(\{z\})=\{y:(y, z) \in \mu(u)\} \\
& =\left\{y:(y, z) \in \Pi \mu\left(u_{\lambda}\right)\right\}, \text { using }(a), \\
& =n\left\{y:(y, z) \in \mu\left(u_{\lambda}\right)\right\}=n \mu_{u_{\lambda}}(z) .
\end{aligned}
$$

(c) Combining (b) and (5),

$$
(\forall \lambda) \mu_{u_{\lambda}}(z)=\mu\left(F_{\lambda}\right) \Rightarrow \mu_{u}(z)=\mu(F) .
$$

It only remains to show that $F$ is a Cauchy filter in $X$. Let $U \in u$ and select a basic vicinity

$$
V=\left\{(x, y): x-y \in \bigcap_{i=1}^{n}\left\{p_{\lambda_{i}}<\varepsilon_{i}\right\}\right\}
$$

such that $V \subset U$. Since $V_{\lambda_{i}}=\left\{(x, y): x-y \in\left\{p_{\lambda_{i}}<\varepsilon_{i}\right\}\right\}$ is in $u_{\lambda_{i}}$ and $F_{\lambda_{i}}$ is Cauchy in $X_{\lambda_{i}}$, we can find $F_{i}$ in $F_{\lambda_{i}}$ such that $F_{i} \times F_{i} \subset V_{\lambda_{i}}$. Put $F=\bigcap_{i=1}^{n} F_{i}$. Then $F \in F$, and if $x, y \in F$ then $x, y \in F_{i}$ for all $i$ and so $(x, y) \in V_{\lambda_{i}}$. Thus $(x, y) \in \cap V_{\lambda_{i}}=V$ and $F \times F \subset V$. It follows that $F$ is Cauchy, and the theorem is proved.

Proof of Theorem 2.1. Assume $z$ is a finite point of ${ }^{*} X$ such that for every equicontinuous subset $S^{\prime}$ of $X^{\prime}, z^{\prime} \in{ }^{*} S^{\prime} \cap \mu_{\sigma\left(X^{\prime}, X\right)}(0)$ implies $z^{\prime}(z)={ }_{1} 0$. Fix $\lambda$ and take $S^{\prime}=S_{\lambda}^{\prime}$. By Lemma 2.10, $S^{\prime}$ is an equicontinuous set. Since $S_{\lambda}^{\prime} \subset X_{\lambda}^{\prime}$, the topologies $\sigma\left(X^{\prime}, X\right)$ and $\sigma\left(X_{\lambda}^{\prime}, X\right)$ coincide on $S_{\lambda}^{\prime}$. Hence

$$
z^{\prime} \in{ }^{*} S_{\lambda}^{\prime} \cap \mu_{\sigma\left(X_{\lambda}^{\prime}, X\right)}(0)=z^{\prime} \in{ }^{*} S^{\prime} \cap \mu_{\sigma\left(X^{\prime}, X\right)}(0)=z^{\prime}(z)=10
$$

by hypothesis. By $([2], 3.17 .2,(c)=(a)), z$ is pre-near-standard in ${ }^{*} X_{\lambda}$. By Theorem 2.11, this proves that $z$ is pre-near-standard in ${ }^{*} X$.

Conversely, let $z$ be a pre-near-standard point of ${ }^{*} X$. Then $z$ is finite, by Corollary 2.3. Let $S^{\prime}$ be an equicontinuous subset of $X^{\prime}$ and 
$z^{\prime} \in{ }^{*} \cap \cap \mu_{\sigma\left(X^{\prime}, X\right)}(0)$. By Theorem 2.7, st ${ }_{\omega}(z)$ is $\sigma\left(X^{\prime}, X\right)$ continuous on $S^{\prime}$, and $s t_{w} z\left(z^{\prime}\right)=z_{1} z^{\prime}(z)$ for $z^{\prime} \epsilon S^{\prime}$ by Theorem 2.8. Hence applying Proposition 2.9, $z^{\prime}(z)=10 \quad\left(z^{\prime} \in{ }^{*} S^{\prime}\right)$.

The proof of Theorem 2.1 is now complete.

\section{References}

[1] Gottfried Köthe, Topological vector spaces I (Translated by D.J.H.

Garling. Die Grundlehren der mathematischen Wissenschaften, Band 159. Springer-Verleg, Berlin, Heidelberg, New York, 1969).

[2] W.A.J. Luxemburg, "A general theory of monads", Applications of model theory to algebra, analysis, and probability (Internat. Sympos., Pasadena, California, 1967, 18-86). (Holt, Rinehart and Winston, New York, Chicago, San Francisco, Atlanta, Dallas, Montreal, Toronto, London, Sydney, 1969).

[3] Moshé Machover, Joram Hirschfeld, Lectures on non-standard analysis (Lecture Notes in Mathematics, 94. Springer-Verlag, Berlin, Heidelberg, New York, 1969).

[4] Abraham Robinson, Non-standard analysis (Studies in Logic and the Foundations of Mathematics. North-Holland, Amsterdam, 1966).

Department of Mathematics, Institute of Advanced Studies, Australian National University, Canberra, ACT. 